\title{
Uterine Perforation with Intra-Abdominal Clostridium perfringens Gas Gangrene: A Rare and Fatal Infection
}

\author{
David Kashan, MD, Nagarajan Muthu, MD, Benjamin Chaucer, MD(cand), Fidencio Davalos, MD, ${ }^{3}$ \\ Michael Bernstein, $\mathrm{MD}{ }^{3}$ and Akella Chendrasekhar, $\mathrm{MD}^{3}$
}

\begin{abstract}
Background: Clostridium perfringens gas gangrene is an extremely rare and fatal infection. Necrosis of the myometrium is rarely seen and has only been recorded in 18 cases to date. Of these 18 reported cases, only 5 have occurred in nonpregnant women. This article presents the 6th case of myometrium necrosis from $C$. perfringens. Case: A 72-year-old woman, gravida 2, para 2, presented with abdominal pain and vaginal bleeding. After examinations, laboratory testing, and several surgical interventions, she was found to have C. perfringens infection and advanced high-grade serous adenocarcinoma of the endometrium with $>50 \%$ invasion into the myometrium. $R \boldsymbol{e}$ sults: Despite the surgical interventions and use of several antibiotics, this patient did not improve. She was weaned from treatment per her advance directive and died after weaning. Conclusions: Awareness of the many etiologies for peritonitis is of great importance when a fatal infection may be the cause of the condition. Correct diagnosis and proper treatment is essential for the survival of patients infected with C. perfringens. ( J GYNECOL SURG 32:182)
\end{abstract}

\section{Introduction}

$\mathbf{T}$ HE PRESENTATION of abdominal pain and peritonitis is common in modern medicine. The common differentials for this presentation include: ruptured appendix; perforated gastric ulcer; diverticulosis; and pancreatitis. The presentation of peritonitis when combined with vaginal bleeding yields a high index of suspicion for a widespread abdominal infection. A widespread abdominal infection may have many infectious etiologies, with some being more common then others. Intra-abdominal Clostridium perfringens gas gangrene infection is extremely rare. It is associated with uterine malignancy and should be considered in gynecologic patients in whom a common etiology cannot de diagnosed. Prompt and aggressive treatment is essential because of the severity of this infection. This article presents the case of a 72-yearold woman with clostridial sepsis and discusses the treatment options for this fatal infection.

\section{Case}

A 72-year-old woman, gravida 2, para 2, with a past medical history of hypothyroidism, hyperlipidemia, hypertension, and osteoarthritis, presented to the emergency room for severe abdominal pain and vaginal bleeding that had begun 8 hours prior to her presentation. Her past surgical history included a bilateral oophorectomy at age 32 for ovarian cysts and a bilateral knee replacements. She has also undergone a colonoscopy, 20 years prior, with benign findings.

In the emergency department, this patient reported severe nausea and vomiting. She had experienced chills since earlier that day, but denied having a fever. She denied either changes in her bowel habits or any pain when urinating. Initial vitals obtained in the emergency department were as follows: temperature: $98.4^{\circ} \mathrm{F}$; blood pressure: $128 / 84 \mathrm{mmHg}$; heart rate: 104 beats per minute; respiration rate: 20 breaths per minute; and $\mathrm{O}_{2}$ saturation of $98 \%$ on room air. She was in severe distress because of her abdominal pain. A physical examination revealed that she had a tympanic, distended abdomen that was tender to palpation and that had rebound tenderness. A complete blood count revealed moderate leukocytosis $\left(12600 / \mathrm{mm}^{3}\right)$; a comprehensive metabolic panel showed an anion gap metabolic acidosis (bicarbonate of 15) and azotemia (blood-urea-nitrogen level of 26 and creatinine level of $2.6 \mathrm{mg} / \mathrm{dL})$.

\footnotetext{
${ }^{1}$ State University of New York Downstate Medical Center, Staten Island, NY.

${ }^{2}$ St. George's University, St. George's, Grenada, West Indies.

${ }^{3}$ Richmond University Medical Center, Staten Island, NY.
}

(C) David Kashan et al. 2015; Published by Mary Ann Liebert, Inc. This Open Access article is distributed under the terms of the Creative Commons Attribution Noncommercial License (http://creativecommons.org/licenses/by-nc/4.0/) which permits any noncommercial use, distribution, and reproduction in any medium, provided the original author(s) and the source are credited. 
Although she was catheterized with a Foley catheter, while she was in the emergency department, her urine output was minimal. She was given $2 \mathrm{~L}$ of normal saline. The gynecology department was consulted and a further examination revealed that she had uterine prolapse and confirmed mild vaginal bleeding. A computed tomography (CT) scan of her abdomen and pelvis with contrast was obtained (Fig. 1).

Pneumoperitoneum and pneumobilia were noted. A large masslike collection of mottled air and soft-tissue density in the area surrounding the uterus was noted, suggesting intrauterine infection or necrosis. The CT scan revealed colonic perforation with a possible enterofistula connecting to the uterus. No evidence of obstruction or other signs of inflammatory disease were noted. The patient was taken to the operating room (OR) for an emergent exploratory laparotomy. Upon entry into the abdominal cavity, there was a burst of foul-smelling gas. There was a $2.5-\mathrm{cm}$ spontaneous rupture of the posterior fundus. An intrauterine malignancy was suspected and a subsequent, emergent total abdominal hysterectomy and salpingectomy were performed. A mass was noted on the distal appendix and, subsequently, an appendectomy was performed. The small bowel and colon were examined. On initial examination, the small bowel and colon appeared to be healthy. The peritoneal cavity was copiously irrigated with normal saline and an antibiotic solution.

Peritoneal-cavity fluid cultures were obtained, and uterine myometrial tissue was sent for pathology assessment. The patient's abdomen was closed with retention sutures and a Jackson-Pratt drain was placed. Despite fluid resuscitation prior to and during the operation, severe end organ damage ensued, which resulted in multi end organ failure. The patient remained intubated and was transferred in critical condition to the surgical intensive care unit.

The pathologic report of the uterine tissue culture indicated advanced high-grade serous adenocarcinoma of the endometrium with $>50 \%$ invasion into the myometrium. Blood gram stain showed large gram-positive rods and the presence of $C$. perfringens was confirmed by blood and intraoperative wound cultures.

On postoperative day (POD) 0, the patient was severely acidemic and was found to be hypotensive. Norepinephrine was administered for pressure support. She was started on broad-spectrum antibiotics, including piperacillin/tazobactam, vancomycin, and metronidazole. On POD 1, the blood and peritoneal fluid cultures returned were positive for $C$. perfringens and her antibiotic regimen was subsequently changed
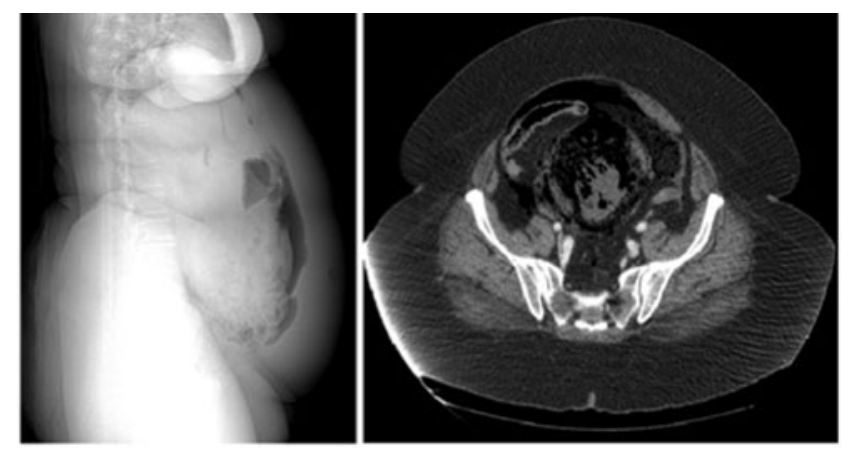

FIG. 1. Computed tomography scan showing free intraabdominal air and uterine myonecrosis. to vancomycin and intravenous (I.V.) penicillin G. On POD 4, the patient's abdomen was noted to be increasingly tense and distended. She had worsening multiorgan failure secondary to sepsis. She was returned to the OR for a reexploration of the abdominal cavity. A large quantity of brown and "ashy" fluid in the peritoneal cavity was removed during this surgery. Upon further exploration, it was noted that there was necrosis of the distal half of the small bowel and the entire colon, including the proximal rectum. Ileectomy, total colectomy, and resection of the proximal rectum were performed. After completing a washout of the peritoneal cavity, the bowel was left in discontinuity with a wound vacuum in place.

\section{Results}

After the surgery, the patient remained in critical condition in the intensive care unit. On POD 6, the patient was taken once again to the OR for ileostomy creation. Focal areas of patchy necrosis were seen at 5 different sites of the small bowel. Because of the complexity of the infection, contiguous resection was not possible. Multiple resections were made and an ileostomy was created. Postoperative follow-up showed necrosis at the site of the ileostomy. As a result of the patient's increasing hemodynamic instability, her advance directives were revisited with the family. Comfort care with terminal wean was initiated in accordance with the family's wishes. The patient died shortly after commencing terminal weaning.

\section{Discussion}

To date, only 18 cases of uterine infected clostridia sepsis have been documented. Of those documented, only 5 were reported in women who were not pregnant. ${ }^{1}$ This case represents the sixth documented case of clostridiainduced necrosis of the myometrium in a nonpregnant female. C. perfringens is an anaerobic gram-positive, nonmotile bacillus found in the normal intestinal and vaginal flora of $1 \%-27 \%$ of healthy women. ${ }^{1}$ This bacterium is often encapsulated and is capable of producing numerous toxins. The bacterial diversity of this pathogen is remarkable, with more than 640 different strains of C. perfringens identified. Only $5 \%$ of these 640 strains are known to be pathogenic. Among the toxins produced is lecithinase $\mathrm{C}$, which is responsible for the hemolysis seen in clostridial infections. ${ }^{2}$

Clostridia infections rarely result in septicemia. When septicemia ensues, the clinical presentation is shock, acute hemolysis, rapidly developing jaundice, tachycardia, hypotension, and often death. The onset of symptoms of clostridia infection is of particular diagnostic interest. Symptoms from clostridia infection have a particularly rapid onset, compared to other infectious causes. Lecithinase $\mathrm{C}$ toxin is responsible for the rapid onset of symptoms that can be seen as soon as 1224 hours after infection. ${ }^{2}$

Uterine clostridia infection should be considered in a patient presenting with extreme uterine pain and symptoms of shock, without any clear cardiac or pulmonary etiology. When septicemia occurs, rapid onset of symptoms with sepsis and hemolysis ending in end organ damage is commonly seen. Thrombophlebitis has been documented as a complication, but this is only seen in extremely rare circumstances and usually occurs as a co-infection. ${ }^{3}$ 
The presence of peritonitis should alert practitioners that the infection is likely to have spread beyond the uterus. Bacteremia and sepsis from $C$. perfringens is very rare and is associated primarily with Cesarean deliveries, in molar pregnancies, and in patients with underlying malignancies., When fulminant sepsis ensues the mortality rate is as high as $85 \%$. Disruption of normal tissue planes and boundaries by invasive gynecologic malignancies is required for clostridia dissemination and the development of tissue necrosis and sepsis.

In the presence of gynecologic malignancies, such as endometrial cancer, the rapid turnover of tumor cells has been hypothesized to create an ischemic and hypoxic milieu necessary for the growth of Clostridium and spore germination. ${ }^{5}$ Russell established a theoretical method of spread of infection to the uterus. ${ }^{6}$ First, the infection requires introduction of the bacteria from either an external source or from normal flora (in a rare subset of women). Second, necrotic tissue must be present within the uterus for an extended period to allow for incubation of the bacteria. Third, the damaged uterine tissue must be exposed to the bacterial pathogen. This route of infection explains why this infection is so rarely seen in nonpregnant patients.

C. perfringens septicemia can lead to the development of gas gangrene, which carries a very high mortality and requires prompt reorganization and treatment. Surgery to debride dead tissue and achieve source control is essential for survival. Aggressive fluid resuscitation and appropriate I.V. antibiotic therapy is required in tandem with surgical intervention. ${ }^{1}$ Proper antibiotic therapy includes the use of penicillin and ampicillin. However, antibiotic treatment is often insufficient as a sole method of treatment. ${ }^{1}$ In most documented cases, antibiotic treatment was used as an adjuvant to surgical debridement. When the foci of the infection are unknown, surgical debridement is required to remove affected tissue and to determine the extent of the infection. Surgical exploration is also required when there is ambiguity about to the extent of necrosis, to remove the affected tissue, and to assess the severity of the infection accurately.

While an antitoxin is available, there is ambiguity regarding the effectiveness of its use. ${ }^{7}$ Early administration of an antitoxin may help prophylaxis, but this has not been clearly demonstrated and requires immediate diagnosis of the correct pathogen. The use of hyperbaric oxygen (HBO) treatment may help as an adjuvant therapy to surgery. Given the extent of the necrosis and the spread of the current patients' infection she was not a candidate for HBO treatment. Its use alone is insufficient unless the infected area is an open wound and the extent of the infection is known. HBO therapy can be used prior to and after surgical treatment. ${ }^{8}$ Its use has been shown to decrease toxemia and produce improvement within 6 hours of treatment.

\section{Conclusions}

The use of emergent surgery and aggressive antibiotics can decrease the mortality seen from clostridial sepsis. ${ }^{9}$ Because of the fatality associated with this rare infection, proper diagnosis and prompt treatment is essential for correct management of patients with this infection.

\section{Acknowledgments}

The current authors acknowledge and thank Richmond University Medical Center and State University of New York Downstate Medical Center for their support in writing this case report.

\section{Author Disclosure Statement}

No competing financial conflicts exist.

\section{References}

1. Kurashina R, Shimada H, Matsushima T, Doi D, Asakura H, Takeshita T. Spontaneous uterine perforation due to clostridial gas gangrene associated with endometrial carcinoma. J Nippon Med Sch 2010;77:166.

2. Nakamura M, Cross WR. The lecithinase (alpha toxin) activity of strains of Clostridium perfringens. Proc Soc Exp Biol Med 1968;127:719.

3. Larson CM, Bubrick MP, Jacobs DM, West MA. Malignancy, mortality, and medicosurgical management of Clostridium septicum infection. Surgery 1995;118:592.

4. Lee CH, Hsieh SY. Case report: Clostridium septicum infection presenting as liver abscess in a case of choriocarcinoma with liver metastasis. J Gastroenterol Hepatol 1999;14:1227.

5. Browne JT, Van Derhor AH, McConnell TS, Wiggins JW. Clostridium perfringens myometritis complicating cesarean section: Report of 2 cases. Obstet Gynecol 1966;28:64.

6. Russell AM. The significance of Clostridium welchii in the cervical swab and blood serum in the post-partum and postabortum sepis. J Obstet Gynaec Brit Emp 1949;56:247.

7. Myers RAM. Hyperbaric oxygen therapy in clostridial infection. South Med J 1985;78:227.

8. Hanson GC, Slack WK, Chew HE, Thomas DA. Clostridal infection of the uterus: A review treatment with hyperbaric oxygen. Postgrad Med J 1966;42:499.

9. Stroumsa D, Ben-David E, Hiller N, Hochner-Celniker D. Severe clostridial pyomyoma following an abortion does not always require surgical intervention. Case Rep Obstet Gynecol 2011;2011:364641.

Address correspondence to: Benjamin Chaucer, $M D($ cand $)$ 400 Forest Avenue, Apt 2R Staten Island, NY 10310

E-mail: benchaucer@gmail.com 